CLINICAL STUDY

\title{
Long-term analysis of the efficacy and tolerability of sorafenib in advanced radio-iodine refractory differentiated thyroid carcinoma: final results of a phase II trial
}

\author{
T C Schneider ${ }^{1}$, R M Abdulrahman ${ }^{2}$, E P Corssmit ${ }^{2}$, H Morreau $^{3}$, J W A Smit ${ }^{2}$ and E Kapiteijn ${ }^{1}$ \\ Departments of ${ }^{1}$ Clinical Oncology, ${ }^{2}$ Endocrinology and Metabolic Diseases and ${ }^{3}$ Pathology, Leiden University Medical Center, PO Box 9600 , \\ 2300 RC Leiden, The Netherlands \\ (Correspondence should be addressed to E Kapiteijn; Email: h.w.kapiteijn@lumc.nl)
}

\begin{abstract}
Objective: We conducted a prospective phase II clinical trial to determine the efficacy of sorafenib in patients with advanced radio-iodine refractory differentiated thyroid cancer. In this article, the long-term results are presented.

Patients and methods: Thirty-one patients with progressive metastatic or locally advanced radioactive iodine refractory differentiated thyroid cancer received sorafenib $400 \mathrm{mg}$ orally twice daily. The study end points included response rate, progression-free survival (PFS), overall survival (OS), best response by Response Evaluation Criteria in Solid Tumors criteria 1.0, and toxicity.

Results: Median PFS was 18 months (95\% confidence interval (95\% CI): 7-29 months) and median OS was 34.5 months (95\% CI: 19-50 months). Eight patients (31\%) achieved a partial response and 11 patients $(42 \%)$ showed stable disease after a median follow-up of 25 months (range 3.5-39 months). Toxicity mostly included hand foot syndrome, weight loss, diarrhea, and rash.

Conclusion: Sorafenib has clinically relevant antitumor activity in patients with progressive metastatic or locally advanced radio-iodine refractory differentiated thyroid cancer. Sorafenib can nowadays be considered as the standard option in these patients.
\end{abstract}

European Journal of Endocrinology 167 643-650

\section{Introduction}

Thyroid cancer is the most prevalent endocrine malignancy, accounting for $95 \%$ of cancers of the endocrine system and $66 \%$ of endocrine cancer mortality in 2010 (1). The current incidence in Europe is $49 / 1000000$ per year, with a nearly three times higher incidence in women (2).

Differentiated thyroid carcinoma (DTC) is by far the most common (95\%) subtype and includes papillary thyroid carcinoma (PTC, 80\%) and follicular thyroid carcinoma (FTC, 10-15\%) as well as subtypes like Hurthle cell carcinomas. The majority of DTCs are slowly progressive, and, when identified at an early stage, frequently cured with adequate surgical management and radioactive iodine 131-I (RAI) ablation therapy. However, metastatic DTC that has become inoperable or refractory to RAI therapy is associated with a less favorable prognosis, as 10-year survival varies between 25 and $40 \%(3,4)$. The efficacy of conventional chemotherapy in DTC is negligible, and chemotherapy is therefore no longer recommended in international guidelines $(5,6)$. As a result of increased understanding of thyroid tumorigenesis, potential targets, and novel therapeutic agents that target biological abnormalities have been identified.

In DTC, the role of activated genetic aberrations in the RET-RAS-RAF-MAPK signaling pathway in tumor development and progression has been determined. The B-type Raf kinase (BRAF) V600E mutation has been reported in $29-69 \%$ of PTC and is associated with recurrent and persistent disease with a higher rate of lymph node metastasis and higher TNM stage $(7,8)$. In a review of 11 studies in thyroid cancer, up to $50 \%$ of follicular and $12 \%$ of Hurthle cell malignancies harbored RAS mutations or RAS downstream signaling PIK3CA mutations (9). Rearrangements in the RET proto-oncogene occur in up to $25 \%$ of PTC, resulting in the generation of chimeric oncogenes responsible for the initiation of tumor formation (10). Additionally, RAS mutations and RET/PTC translocations also result in aberrant signaling through BRAF. Furthermore, the RET-RAS-RAF pathway leads to vascular endothelial growth factor (VEGF) and VEGF receptor (VEGFR) synthesis through interconnection with the epithelial growth factor receptor (EGFR)-activated cascade (11). In turn, these are the most important regulators in the process of angiogenesis. Increased vascularity plays 
a crucial role in tumor growth and generating metastasis (12). In human thyroid cancer, VEGF overexpression is associated with increased tumor stage and invasiveness (11). Pazopanib is a tyrosine kinase inhibitor (TKI) that targets VEGFR1-3 and c-KIT. Its antitumor activity in advanced and progressive DTC was demonstrated in a phase II trial, which showed a partial response $(\mathrm{PR})$ rate in $49 \%$ of patients $(n=39)$. Median progression-free survival (PFS) was 12 months $(13,14)$. XL184 (cabozantinib) is an oral inhibitor of RET, c-MET, and VEGFR1 and 2. c-MET activation triggers tumor growth and angiogenesis. Moreover, in patients with PTC and MTC, overexpression and frequent mutations of the c-MET receptor have been reported (15). Antitumor activity of cabozantinib in patients with DTC was recently reported at the 2012 ASCO Annual Meeting. Cabanillas et al. found confirmed PR and stable disease (SD) in 53 and $40 \%$ of patients respectively $(n=15)$. Disease control rate $(\mathrm{PR}+\mathrm{SD})$ was $80 \%$ at 16 weeks. At the time of data presentation, median PFS and overall survival (OS) had not been reached (16).

E7080 (lenvatinib) is also an inhibitor of multiple TK, especially VEGFRs, c-KIT, and platelet-derived growth factor receptor $\beta$ stem cell factor receptor. In animal studies, it has been shown to have potent antitumor activity against small-cell lung cancer and breast cancer most likely through inhibition of VEGFR2 and $3(17,18)$. A phase II trial of lenvatinib in RAI refractory DTC $(n=58)$ by Sherman et al. (19) showed a PR rate of $50 \%$ and a median PFS of 13 months. A recent study by Rajoria et al. (20) showed estrogen to be a key regulating factor of VEGF expression in thyroid cells, suggesting a role for antiestrogens in the therapeutic regimen to treat thyroid cancer.

As a result of this increased understanding of the biological basis for thyroid cancer development, multiple clinical trials with multi-target TKIs have been conducted. Sorafenib (BAY 43-9006) is an orally active TKI that targets BRAF, VEGFR1 and 2, and RET, implementing antiangiogenic and proapoptotic actions. Results of an update of a phase II study on sorafenib, reported at the 2011 ASCO Annual Meeting by Brose et al., showed a PFS of 24 months and an OS of 35 months in 47 patients with DTC and poorly differentiated thyroid cancer (PD; $n=55)$. A PR was achieved in $38 \%$ of patients and $47 \%$ had SD (21). Another phase II study in mainly patients with advanced DTC $(n=30)$ reported a PR rate of $23 \%$, an SD rate of $53 \%$, and a median PFS of 20 months (22). Kloos et al. examined the effect of sorafenib mainly in patients with metastatic PTC $(n=41)$ and reported a PR and SD of 15 and $56 \%$ respectively. The median PFS was 15 months (23). Recent results of a study with the MEK $1 / 2$ inhibitor selumetinib showed reinduction of radio-iodine uptake in $60 \%$ of patients with RAI refractory thyroid cancers of follicular origin (24).
Our phase II study was designed to assess the effects of sorafenib on restoring the susceptibility to RAI therapy, tumor progression, and safety in RAI refractory DTC. The first report of this study by Hoftijzer et al. (25) showed a promising efficacy and tolerability of sorafenib, but no beneficial effects on radio-iodine uptake. Here, we report the long-term outcomes of this sorafenib trial in advanced RAI refractory DTC with results on efficacy, safety, and tolerability.

\section{Materials and methods}

\section{Patients}

Details of study design and patient eligibility of this study have been described previously (25). Briefly, eligibility criteria were the presence of progressive metastases or unresectable local recurrence of DTC for which RAI therapy was no longer effective, as indicated by prior negative post-therapeutic whole-body scintigraphy. Progressive disease was defined according to Response Evaluation Criteria in Solid Tumors (RECIST) criteria 1.012 months before initiation of treatment. Patients had undergone total thyroidectomy and RAI ablative therapy. Eastern Cooperative Oncology Group performance status had to be $<2$ and life expectancy had to be more than 3 months. Written informed consent was provided by all patients before enrollment onto the trial. The study protocol was approved by the Institutional Review Board of the Leiden University Medical Center. This study was registered at ClinicalTrials.gov (\#NCT00887107).

\section{Study design}

We performed a nonrandomized, open-arm, phase II trial of sorafenib in 31 patients with advanced RAI refractory DTC. For a complete description of study design, we refer to the paper of Hoftijzer et al. (25). The primary objective of this study was to determine the efficacy of sorafenib treatment with a PR as the primary end point. The secondary objective was to study RAI reuptake and the safety of sorafenib as an antitumor drug for advanced RAI refractory DTC patients. Sorafenib was administered at a dose of $400 \mathrm{mg}$ orally twice a day until disease progression, uncontrollable side effects, death, or patients' own request. Safety assessments consisted of physical examination, documentation of adverse events (AEs), and laboratory parameters (total blood count, electrolytes, and kidney and liver function) and were performed every 4 weeks. The incidence, grade, and relationship of AEs to the drug were graded with the use of Common Terminology Criteria for Adverse Events (CTCAE, version 3.0).

After completion of 6-month treatment, patients were allowed to continue sorafenib treatment when a favorable response (complete remission, partial 


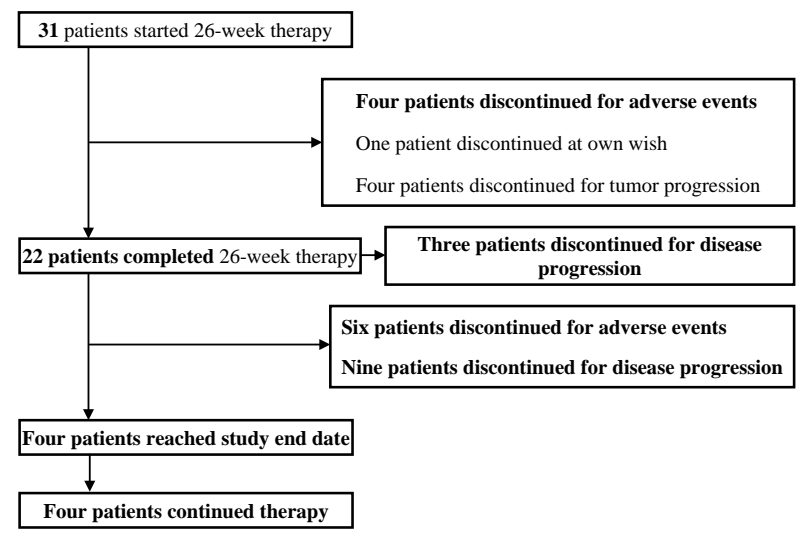

Figure 1 Study flowchart.

remission, or SD) had been achieved. Sorafenib was thereafter continued until progression according to RECIST 1.0 criteria with computed tomography (CT) scanning performed every 6 months. In this article, we report the long-term outcomes and tolerability of sorafenib in patients with advanced RAI refractory DTC.

\section{Statistical analysis}

In this study, data are reported as median \pm s.D., median (range), or proportions. Best objective response rate (RR) refers to the proportion of patients who had the best response rating for complete response (CR) or PR according to RECIST 1.0. The OS, PFS (defined as the time from starting study drug to progression), and overall disease control rate with associated 95\% confidence intervals (95\% CIs) were obtained using the Kaplan-Meier method. AEs were scored according to the National Cancer Institute's CTCAE, version 3.0. Other safety parameters, including laboratory data, were summarized descriptively. Variables possibly influencing response to sorafenib were analyzed with binomial logistic regression. The calculations were performed using SPSS 17.0 for Windows (SPSS, Chicago, IL, USA).

\section{Results}

\section{Patient characteristics}

All 31 patients were included in this long-term outcome study on sorafenib. The follow-up of the study ended on 1 March 2011 with five patients excluded from the efficacy analysis because they did not reach the first radiological evaluation at 6 months. One patient requested to stop sorafenib treatment after 4 days, one patient developed small-cell lung cancer, and three patients discontinued treatment because of AEs. Median follow-up period was 25 months (range 3.5-39 months) and median sorafenib treatment period was
9.2 months (range 0.1-39 months). By the time the follow-up of the study ended (March 2011), four patients $(15.4 \%)$ were still on sorafenib (Fig. 1).

Baseline characteristics of the included patients are listed in Table 1. As can be expected, Hurthle cell metaplasia, which is associated with poor RAI responsiveness, was overrepresented in the patient group $(42 \%)$. Two of 13 PTC patients had a poorly differentiated carcinoma. A total of 15 mutations were identified on primary tumor material, of which BRAF V600E was most frequently observed with 10/13 PTC patients harboring a BRAF V600E mutation.

\section{Efficacy}

Of the 31 patients enrolled in this study, 26 patients were eligible for efficacy analysis. Five patients were excluded for the reasons previously mentioned. Data on efficacy are given in Table 2 and Fig. 2.

At a median follow-up of 25 months, the overall disease control rate was $27 \%$. The total proportion of PR was $31 \%(n=8)$, which were all achieved in the first 6 months of treatment. Four patients $(15 \%)$ had an

Table 1 Baseline characteristics.

\begin{tabular}{ll}
\hline & All patients \\
& $(n=31)$ \\
\hline Gender ( $n$, \%) & \\
Female & $12(39)$ \\
Male & $19(61)$ \\
Age (year; median, range) & $64(53-82)$ \\
Time from diagnosis (year; median, range) & $3.9(0.3-18)$ \\
Histology ( $n$, \%) & \\
Papillary & $13(42)$ \\
Tall cell & $1(3)$ \\
Hurthle cell metaplasia & $2(7)$ \\
Poorly differentiated & $2(7)$ \\
Follicular variant papillary & $2(7)$ \\
Follicular & $15(48)$ \\
Hurthle cell metaplasia & $11(35)$ \\
Mixed papillary follicular & $1(3)$ \\
Initial TNM stage ( $n$, \%) & \\
IB (T2NOM0) & $2(7)$ \\
IIB (T2-3 N0-1 M0) & $16(52)$ \\
IIIA (T1-3 N1-2 M0) & $5(16)$ \\
IV (any T any N M1) & $6(19)$ \\
Unknown & $2(7)$ \\
Tumor extent at study entry ( $n$, \%) & $1(3)$ \\
Thyroid bed only & $8(26)$ \\
Lungs only & $8(26)$ \\
Lungs and bone only & $9(29)$ \\
Locally advanced a and distant metastases & $5(16)$ \\
Other & \\
Mutational status ( $n$, \%) & $10(32)$ \\
$B R A F$ & $1(3)$ \\
K-RAS & $2(6)$ \\
$N-R A S$ & 0 \\
$H-R A S$ & $2(6)$ \\
$P I K 3 C A$ & \\
\hline
\end{tabular}

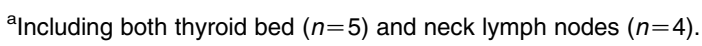


Table 2 Efficacy analysis

\begin{tabular}{ll}
\hline & $\begin{array}{l}\text { No. of } \\
\text { patients } \\
(\% \text { of total) }\end{array}$ \\
Parameter & 31 \\
\hline Total patients & 26 \\
Assessable patients & $15(0.1-39)$ \\
Median duration of treatment (months, range) & $25(3.5-39)$ \\
Median duration of follow-up (months, range) & \\
Best response by RECIST 1.0 & $0(0)$ \\
Complete response & $8(31)^{\mathrm{a}}$ \\
Partial response & $3(12)$ \\
Stable disease & $15(58)$ \\
Progressive disease & $7(27)$ \\
Overall disease control & $26.5(3-38)$ \\
Median duration of SD (months, range) & $29.6(3-33)$ \\
Median overall response duration (months, range) & $18(7-29)$ \\
Median PFS (95\% CI) & $12(\mathrm{NR})$ \\
With BM & $20(13-27)$ \\
Without BM & $34.5(19-50)$ \\
Median OS (95\% CI) & $23(20-26)$ \\
With BM & $\mathrm{NR}$ \\
Without BM &
\end{tabular}

RECIST, Response Evaluation Criteria in Solid Tumors; SD, stable disease; PFS, progression-free survival; OS, overall survival; BM, bone metastases; $\mathrm{NR}$, not reached.

${ }^{\text {a }}$ Four of eight patients showed an ongoing PR.

ongoing PR and three patients (12\%) showed ongoing SD. There were no CRs. Individual data per patient response is summarized in Table 3. At the time of this data analysis, the cumulative number of patients with progressive disease was 15 (58\%). Disease progression was not influenced by age, gender, or histology, including the presence of Hurthle cell metaplasia. Furthermore, the presence of a BRAF V600E mutation was not related to disease progression. The prevalence of other mutations was too low to allow statistical analysis. Disease progression in soft tissue metastases or bone metastases did not significantly differ. However, the favorable responses seen in three patients with bone metastases were based on regression of metastases other than bone, predominantly lung, whereas the bone metastases were stable. The overall median OS was 34.5 months (95\% CI: $19-50$ months). In the presence of bone metastases, median OS was significantly worse with 23 months (95\% CI: 20-26 months) compared with the median OS of the whole group. However, at the time of this data analysis, median OS was not reached for patients without bone metastases (Fig. 2A). Age and gender did not influence OS. Estimation of median PFS was 18 months $(95 \%$ CI: 7-29 months). Similarly, the median PFS was influenced by the presence of bone metastases. Median PFS was 20 and 12 months in the absence and presence of bone metastases respectively (Fig. 2B). A relation between OS and PFS and site of bone metastases could not be established as 12 of 13 patients with bone metastases had metastases localized in the axial skeleton. Hurthle cell metaplasia and reduction of drug dose did not influence PFS.
Thyroglobulin $(\mathrm{Tg})$ response reflected the radiological response (Table 4). From baseline, patients with a PR had a median decrease of $24 \mu \mathrm{g} / \mathrm{l}$ (range, -2746 to $2.6 \mu \mathrm{g} / \mathrm{l}$ ) in their serum Tg levels. Patients with SD showed a median elevation of $19 \mu \mathrm{g} / \mathrm{l}$ (range, -60 to $3724 \mu \mathrm{g} / \mathrm{l})$. The serum $\mathrm{Tg}$ levels in patients that showed PD increased by a median of $49 \mu \mathrm{g} / \mathrm{l}$ (range, -5320 to $87736 \mu \mathrm{g} / \mathrm{l})$. The 54-year-old woman with pulmonary metastases of a Hurthle cell FTC reported at 6 months with high Tg levels $(99900 \mu \mathrm{g} / \mathrm{l})$ but a decrease in number, size, and density of all metastases died 2 months later from disease progression with a $\mathrm{Tg}$ level that remained high $(89400 \mu \mathrm{g} / \mathrm{l})$.
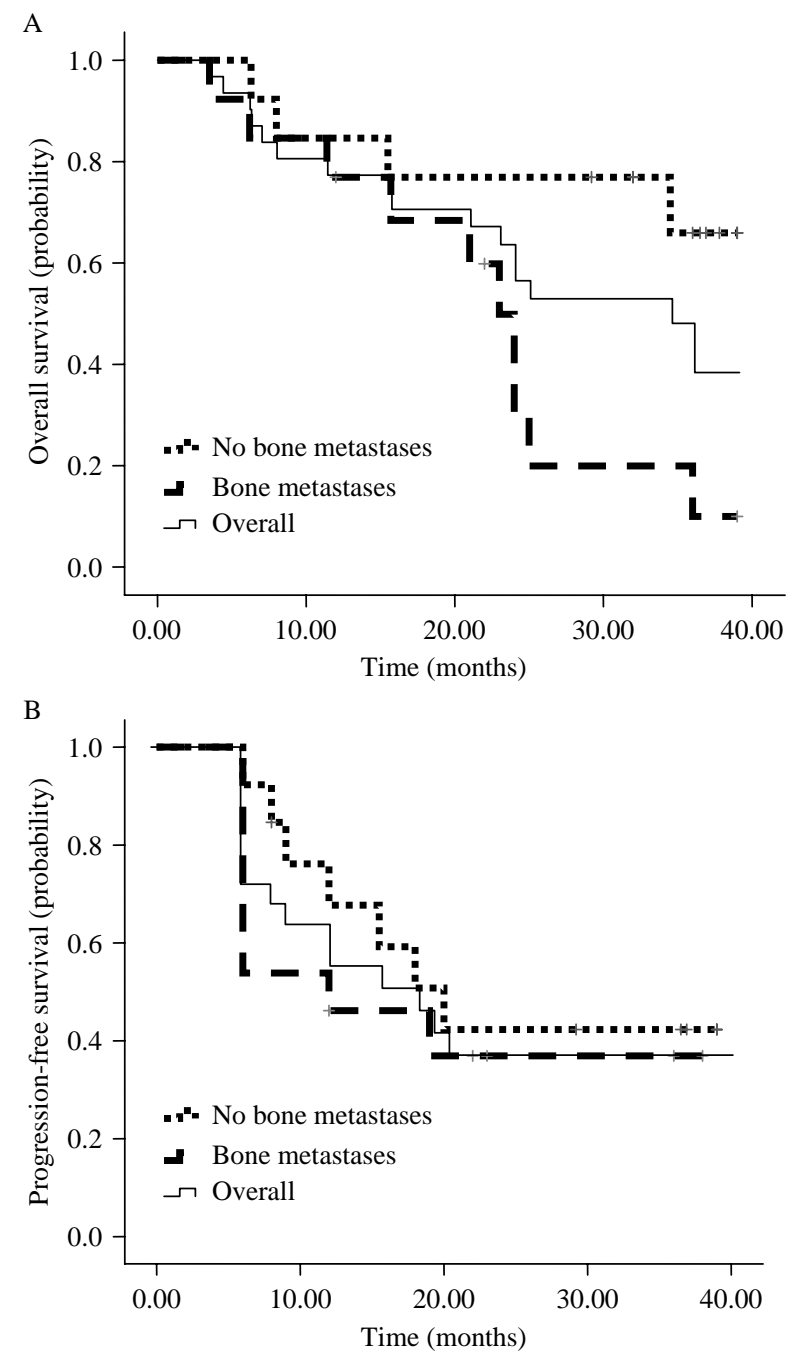

Figure 2 Kaplan-Meier curves of (A) overall survival (OS) and (B) median progression-free survival (PFS) (A) Kaplan-Meier estimate of OS. Overall median OS was 34.5 months. In the presence of bone metastases, median OS was 23 months. At the time of this data analysis, median OS was not reached for patients without bone metastases. (B) Kaplan-Meier estimate of PFS. Estimation of median PFS was 18 months. Median PFS was 20 months in the absence of and 12 months in the presence of bone metastases. 
Table 3 Data on clinical benefit per patient.

\begin{tabular}{llcc}
\hline Response & Histology & $\begin{array}{c}\text { Initial } \\
\text { tumor } \\
\text { stadium }\end{array}$ & $\begin{array}{c}\text { Duration of } \\
\text { response } \\
\text { (months) }\end{array}$ \\
\hline PR & Papillary & IB & $33^{\mathrm{a}}$ \\
PR & Follicular with Hurthle & IIB & $33^{\mathrm{a}}$ \\
PR & cell metaplasia & IIB & $33^{\mathrm{a}}$ \\
PR & Papillary & IV & 30 \\
PR & Papillary & IIB & 24 \\
PR & Follicular & IIB & 24 \\
PR & Papillary & IIB & $18^{\mathrm{a}}$ \\
PR & Papillary & IIIA & 6 \\
SD & Papillary & IIIA & 38 \\
SD & Follicular & IIB & 36 \\
SD & Follicular with Hurthle & IIIB & 12 \\
& cell metaplasia & & \\
\hline
\end{tabular}

PR, partial response; SD, stable disease; IB (T2NOMO); IIB (T2-3 N0-1 MO); IIIA (T1-3 N1-2 M0); IV (any T any N M1).

${ }^{\mathrm{a}}$ Ongoing response.

\section{Treatment tolerability and AEs}

Eighteen patients (58\%) required dose reduction due to toxicity. Four of them (13\%) discontinued the study later on due to AEs. Of these, three patients suffered from a myocardial infarction after 1, 20, and 22 months of sorafenib treatment respectively and one patient quit due to complaints of malaise after 39 months. Forty-four percent of dose reductions occurred in the first month of treatment. Further details on dose reduction and daily sorafenib dose are listed in Table 5. In total, seven patients $(23 \%)$ terminated study participation as a result of drug-related AEs. There were three patients who discontinued sorafenib before dose reduction after 1,12 , and 19 months due to angioedema, congestive heart disease, and malaise and weight loss respectively. One patient discontinued treatment after 3 months due to the development of small-cell lung cancer. One patient was diagnosed with carcinoma of the tongue after 46 months of sorafenib treatment. Thirteen patients $(42 \%)$ required levothyroxine dose adjustments in order to maintain euthyroidism.

All observed AEs are listed in Table 6. Treatmentrelated AEs were predominantly of grade 1 or 2 , with the most common events including hand foot syndrome (HFS), weight loss, diarrhea, and rash. Grade 3 AEs consisted of HFS (23\%), weight loss $(10 \%)$, diarrhea
$(6 \%)$, rash $(16 \%)$, mucositis $(10 \%)$, and congestive heart disease (3\%). Grade 4 AEs comprised myocardial infarction in three patients $(10 \%)$, which led to death within 2 months after the infarction in one patient. The majority of AEs were seen in the first year of treatment and were controllable with dose reduction, medication, or supporting measures (i.e. dietary consultation and additional feeding). No correlations were found between toxicity and performance state or age.

\section{Discussion}

The developments in the treatment of advanced thyroid cancer are a result of increased understanding of thyroid tumorigenesis. A high percentage of differentiated thyroid cancers contain aberrations in the RET/PTC-RAS-RAF-MAPK pathway. In addition, the RET-RAS-RAF pathway leads to VEGF and VEGFR synthesis through the EGFR-activated cascade. Hence, compounds targeting the activated RET-RAS-RAF pathway and beyond, antiangiogenic compounds or a combination of both, may be effective in RAI non-avid DTC (26). Several clinical trials in thyroid cancer have shown considerable percentages of clinical responses, suggesting this assumption to be accurate. Of the targeted therapies that have shown promising results in advanced differentiated thyroid cancer, only sorafenib targets both BRAF and RET, as well as VEGFR. However, sorafenib is a relatively weak BRAF inhibitor. The most potent BRAF inhibitor, vemurafenib, might be more effective in PTC harboring a BRAF mutation and is currently under study in an international phase II trials (www.clinicaltrials.gov, NCT01286753) (27).

Our study is the first study reporting the long-term effects of sorafenib treatment in advanced RAI refractory DTC patients. We also provided results of the influence of bone metastases on survival outcome and PFS. Our results were not influenced by subtypes of thyroid cancer, as we only included patients with DTC, ensuring a homogeneous study population. In our trial, we observed beneficial effects of sorafenib. At 6 and 25 months, median PFS was 14.5 and 18 months respectively. At 6 and 25 months, median OS was 25 months and 34.5 months respectively. The PR rate of $25 \%$ we observed in the first 6 months of the trial is one of the highest PR rates reported in sorafenib trials in DTC. Kloos et al. (23) found a PR rate of $15 \%$ in patients

Table 4 Thyroglobulin response per patient response.

\begin{tabular}{llcll}
\hline RECIST 1.0 & CR & PR & SD & PD \\
\hline Baseline serum Tg levels (median, range) & Non & $50(4.5$ to 3126$)$ & 41 (2 to 1817$)$ & $382(26.5$ to 8570$)$ \\
Last serum Tg levels (median, range) & Non & $19(0.9$ to 1894$)$ & $94(14$ to 4140$)$ & $373(38$ to 89400$)$ \\
Delta Tg (vs baseline; median, range) & Non & $-24(-2746$ to 2.6) & $19(-60.8$ to 3724) & $49(-5320$ to 87 736) \\
\hline
\end{tabular}

RECIST, Response Evaluation Criteria in Solid Tumors; Tg, thyroglobulin; CR, complete response; PR, partial response; SD, stable disease; PD, progressive disease. 
Table 5 Daily sorafenib dose and dose reduction.

\begin{tabular}{llll}
\hline & $\mathbf{3}$ months & $\mathbf{6}$ months & $\mathbf{1 2}$ months \\
\hline $\begin{array}{c}\text { Dose reduction } \\
(n, \%)\end{array}$ & $13(42)$ & $16(52)$ & $18(58)$ \\
$\begin{array}{c}\text { Daily sorafenib } \\
\text { dose (mg) }\end{array}$ & & & \\
$\begin{array}{l}\text { Mean ( } \pm \text { S.D. }) \\
\text { Median (range) }\end{array}$ & $671( \pm 198)$ & $584( \pm 230)$ & $562( \pm 175)$ \\
& $600(200-800)$ & $600(200-800)$ & $600(400-800)$ \\
\hline
\end{tabular}

with metastatic PTC. Gupta-Abramson et al. (22) reported a PR of $23 \%$ (DTC, MTC, and anaplastic thyroid carcinoma (ATC)) whereas Ahmed et al. (28) observed a PR in $21 \%$ of patients with metastatic thyroid cancer (DTC and MTC). Our high PR rate could be attributed to the differences in patient categories (histologies, tumor stages, sites of metastases, and tumor extent), study design, and analytical methods between the different phase II trials.

The explanation for the significantly less favorable outcome in patients with bone metastases remains unclear. The relationship between the genetic profile of bone metastases and responsiveness to sorafenib is hard to establish given the lower tissue levels and difficulties in obtaining tissue from bone metastases. It can be hypothesized that VEGFR-targeted therapies are less effective in bone metastases as the role of VEGFR signal transduction in tumor expansion may differ between soft tissue and bone metastases. Another explanation may be that through systemic release of cytokines or proteins from the bone microenvironment, the presence of bone metastases influences the response to sorafenib in soft tissue metastases. The dose of sorafenib used in our study was generally well tolerated, although dose reductions were required in $58 \%$ of patients. Tumor response, however, was not affected by dose reductions. Toxicities observed in our study were mostly grades 1 and 2 and similar to those reported in the other phase II trials of sorafenib $(21,22,23,28,29)$. A long-term safety evaluation of sorafenib in advanced renal cell carcinoma showed similar results, supporting our findings (30). Dermatological AEs were the most commonly observed. A recent study of sorafenib in patients with hepatocellular carcinoma reported better response in patients with early skin reactions (31); however, we were not able to establish that correlation. Adverse effects were in the majority of cases manageable with dose reduction, medication, or supplementary measures. However, beneficial effects on tumor response should always be weighed against the side effects and quality of life of targeted therapies $(3,4,29)$.

There are some issues that have to be addressed too. RECIST criteria should be applied for judgment of progression for inclusion in trials and for follow-up scans to determine treatment effect. However, RECIST criteria have several limitations for the determination of efficacy of targeted drug activity. This is shown by the fact that in our study, some of the patients with PD based on new lesions had stable or even reduction in the target lesion's longest diameter. Nevertheless, the RECIST criteria are the best criteria to date and should be used in every clinical trial.

Another issue is that in studies of targeted therapy in advanced thyroid cancer, a substantial proportion of

Table 6 Adverse events. According to Common Terminology Criteria for Adverse Events (version 3).

\begin{tabular}{|c|c|c|c|c|c|}
\hline \multirow[b]{2}{*}{ Event } & \multirow{2}{*}{$\begin{array}{c}\text { All } \\
\text { No. of patients } \\
\text { (\% of total }(n=31))\end{array}$} & \multicolumn{4}{|c|}{ Grades No. of patients (\% of category) } \\
\hline & & 1 & 2 & 3 & 4 \\
\hline Hand foot syndrome & $22(71)$ & $8(36)$ & $7(32)$ & $7(32)$ & \\
\hline Weight loss & $18(58)$ & $7(39)$ & $10(56)$ & $3(17)$ & \\
\hline Diarrhea & $16(52)$ & $5(31)$ & $9(56)$ & $2(13)$ & \\
\hline Rash & $17(55)$ & $11(65)$ & $1(6)$ & $5(29)$ & \\
\hline Alopecia & $16(52)$ & $14(88)$ & $2(12)$ & & \\
\hline Mucositis & $15(48)$ & $11(73)$ & $1(7)$ & $3(20)$ & \\
\hline Hypocalcemia & $15(48)$ & $14(93)$ & $1(7)$ & & \\
\hline Hypertension & $13(42)$ & $4(31)$ & $4(31)$ & $5(38)$ & \\
\hline Hypophosphatemia & $11(35)$ & $11(100)$ & & & \\
\hline Anemia & $11(35)$ & $11(100)$ & & & \\
\hline Hypoparathyroidism & $10(32)$ & & $10(100)$ & & \\
\hline Thrombopenia & $9(29)$ & $9(100)$ & & & \\
\hline Hypothyroidism & $8(26)$ & & $8(100)$ & & \\
\hline Leukopenia & $7(23)$ & $7(100)$ & & & \\
\hline Nausea & $3(10)$ & $3(100)$ & & & \\
\hline Myocardial infarction & $3(10)$ & & & & $3(100)$ \\
\hline Congestive heart disease & 1 (3) & & & $1(100)$ & \\
\hline Hematuria & $1(3)$ & & $1(100)$ & & \\
\hline Deep venous thrombose & $1(3)$ & & & $1(100)$ & \\
\hline Hyponatremia & $1(3)$ & $1(100)$ & & & \\
\hline Pneumothorax & $1(3)$ & & $1(100)$ & & \\
\hline Small-cell lung cancer & $1(3)$ & & & & $1(100)$ \\
\hline
\end{tabular}


patients (33-50\%) had SD of varying duration as best response (32). Given the indolent natural history of these tumors, SD may be considered to be of limited value. Considering this, the determination of the best primary end point remains an issue. Objective RRs do not correlate per se with OS. Given the indolent nature of advanced thyroid cancer, PFS and OS are not always preferred primary end points. Therefore, only patients with documented progressive disease should be included in clinical trials, permitting a smaller sample size and a shorter time for follow-up to demonstrate a difference in response to therapy between treatment groups. In addition, sorafenib treatment should only be initiated in patients with progressive disease or significant tumor burden in the presence of RECIST targets. Furthermore, patients with SD at baseline may not benefit from therapy and do not need treatment with targeted agents, with all possible side effects.

In conclusion, sorafenib is generally well tolerated and has demonstrated to be clinically active in the long term in advanced RAI refractory DTC. In order to enhance sorafenib treatment efficacy, further trials need to focus on sequential or combination therapy, as patients eventually become progressive on sorafenib or do not tolerate it. More trials with new targeted agents are underway and the outcomes of these trials will contribute to a better treatment and understanding of advanced thyroid cancer.

\section{Declaration of interest}

The authors declare that there is no conflict of interest that could be perceived as prejudicing the impartiality of the research reported.

\section{Funding}

J W A Smit received a research grant from Bayer BV, Mijdrecht, The Netherlands (IMP 12791 19-04-2007) for performing this study.

\section{References}

1 American Cancer Society. Cancer Facts \& Figures 2010. Atlanta: American Cancer Society; 2010.

2 GLOBOCAN 2008 v2.0, Cancer Incidence and Mortality Worldwide: IARC CancerBase.

3 Schlumberger M. Management of refractory thyroid cancers. Annales d'Endocrinologie 201172 149-157. (doi:10.1016/j.ando. 2011.03.020)

4 Schlumberger MJ. Diagnostic follow-up of well-differentiated thyroid carcinoma: historical perspective and current status. Journal of Endocrinological Investigation 199922 3-7.

5 Cooper DS, Doherty GM, Haugen BR, Kloos RT, Lee SL, Mandel SJ, Mazzaferri EL, McIver B, Sherman SI, Tuttle RM \& American Thyroid Association Guidelines Taskforce. Management guidelines for patients with thyroid nodules and differentiated thyroid cancer. Thyroid 200616 109-142. (doi:10.1089/thy.2006.16.109)

6 Pacini F, Schlumberger M, Dralle H, Elisei R, Smit JW, Wiersinga W \& European Thyroid Cancer Taskforce. European consensus for the management of patients with differentiated thyroid carcinoma of the follicular epithelium. European Journal of Endocrinology 2006 154 787-803. (doi:10.1530/eje.1.02158)
7 Kebebew E, Weng J, Bauer J, Ranvier G, Clark OH, Duh QY, Shibru D, Bastian B \& Griffin A. The prevalence and prognostic value of BRAF mutation in thyroid cancer. Annals of Surgery 2007 246 466-470. (doi:10.1097/SLA.0b013e318148563d)

8 Riesco-Eizaguirre G, Gutierrez-Martinez P, Garcia-Cabezas MA, Nistal M \& Santisteban P. The oncogene BRAF V600E is associated with a high risk of recurrence and less differentiated papillary thyroid carcinoma due to the impairment of $\mathrm{Na}+/ \mathrm{I}-$ targeting to the membrane. Endocrine-Related Cancer 200613 257-269. (doi:10.1677/erc.1.01119)

9 Segev DL, Umbricht C \& Zeiger MA. Molecular pathogenesis of thyroid cancer. Surgical Oncology 200312 69-90. (doi:10.1016/ S0960-7404(03)00037-9)

10 Kimura ET, Nikiforova MN, Zhu Z, Knauf JA, Nikiforov YE \& Fagin JA. High prevalence of BRAF mutations in thyroid cancer: genetic evidence for constitutive activation of the RET/PTC-RASBRAF signaling pathway in papillary thyroid carcinoma. Cancer Research 200363 1454-1457.

11 Espinosa AV, Porchia L \& Ringel MD. Targeting BRAF in thyroid cancer. British Journal of Cancer 200796 16-20. (doi:10.1038/sj. bjc.6603520)

12 Hicklin DJ \& Ellis LM. Role of the vascular endothelial growth factor pathway in tumor growth and angiogenesis. Journal of Clinical Oncology 20053 1011-1027.

13 Bible KC, SMallridge RC \& Maples WJ. Phase II trial of pazopanib in progressive, metastatic, iodine-insensitive differentiated thyroid cancers. Journal of Clinical Oncology 2009273521.

14 Bible KC, Suman VJ \& Molina JR. Efficacy of pazopanib in rapidly progressive radioiodine-refractory metastatic differentiated thyroid cancers: results of a phase 2 consortium study. Lancet Oncology 201011 962-972. (doi:10.1016/S1470-2045(10)70203-5)

15 Ivan M, Bond JA \& Prat M. Activated ras and ret oncogenes induce over-expression of c-met (hepatocyte growth factor receptor) in human thyroid epithelial cells. Oncogene 199714 2417-2423. (doi:10.1038/sj.onc.1201083)

16 Cabanillas ME, Brose MS \& Ramies DA. Anti-tumor Activity Observed in a Cohort of Patients (pts) with Differentiated Thyroid Cancer (DTC) in a Phase 1 Study of Cabozantinib (XL184). American Thyroid Association, 81st Annual Meeting, 10/2011.

17 Matsui J, Yamamoto Y \& Funahashi Y. E7080, a novel inhibitor that targets multiple kinases, has potent antitumor activities against stem cell factor producing human small cell lung cancer H146, based on angiogenesis inhibition. International Journal of Cancer 2008122 664-671. (doi:10.1002/ijc.23131)

18 Matsui J, Funahashi Y \& Uenaka T. Multi-kinase inhibitor E7080 suppresses lymph node and lung metastases of human mammary breast tumor MDA-MB-231 via inhibition of vascular endothelial growth factor-receptor (VEGF-R) 2 and VEGF-R3 kinase. Clinical Cancer Research: an Official Journal of the American Association for Cancer Research 200814 5459-5465. (doi:10. 1158/1078-0432.CCR-07-5270)

19 Sherman SI, Jarzab B \& Cabanillas ME. Phase II, Multicenter, Open-label, Single Arm Trial to Evaluate the Safety and Efficacy of Oral E7080 in Medullary and Iodine-131 Refractory, Unresectable Differentiated Thyroid Cancers, Stratified by Histology.

20 Rajoria S, Suriano R \& George AL. Molecular target based combinational therapeutic approaches in thyroid cancer. Journal of Translational Medicine 201210 81. (doi:10.1186/14795876-10-81)

21 Keefe SM, Troxel AB, Rhee S, Puttaswamy K, O'Dwyer PJ, Loevner LA, Mandel SJ \& Brose MS. Phase II trial of sorafenib in patients with advanced thyroid cancer. Journal of Clinical Oncology 201129.

22 Gupta-Abramson V, Troxel AB, Nellore A, Puttaswamy K, Redlinger M, Ransone K, Mandel SJ, Flaherty KT, Loevner LA, O'Dwyer PJ \& Brose MS. Phase II trial of sorafenib in advanced thyroid cancer. Journal of Clinical Oncology 200826 4714-4719. (doi:10.1200/JCO.2008.16.3279)

23 Kloos RT, Ringel MD, Knopp MV, Hall NC, King M, Stevens R, Liang J, Wakely PE Jr, Vasko VV, Saji M, Rittenberry J, Wei L, Arbogast D, Collamore M, Wright JJ, Grever M \& Shah MH. Phase 
II trial of sorafenib in metastatic thyroid cancer. Journal of Clinical Oncology 200927 1675-1684. (doi:10.1200/JCO.2008.18. 2717)

24 Loh Ho A, Leboeuf R \& Grewal RK. Reacquisition of RAI uptake in RAI-refractory metastatic thyroid cancers by pretreatment with the MEK inhibitor selumetinib. Journal of Clinical Oncology 2012 30 (suppl; abstract 5509).

25 Hoftijzer H, Heemstra KA, Morreau H, Stokkel MP, Corssmit EP, Gelderblom H, Weijers K, Pereira AM, Huijberts M, Kapiteijn E, Romijn JA \& Smit JW. Beneficial effects of sorafenib on tumor progression, but not on radioiodine uptake, in patients with differentiated thyroid carcinoma. European Journal of Endocrinology 2009161 923-931. (doi:10.1530/EJE-09-0702)

26 Knauf JA \& Fagin JA. Role of MAPK pathway oncoproteins in thyroid cancer pathogenesis and as drug targets. Current Opinion in Cell Biology 200921 296-303. (doi:10.1016/j.ceb. 2009.01.013)

27 Xing J, Liu R, Xing M \& Trink B. The BRAFT1799A mutation confers sensitivity of thyroid cancer cells to the BRAFV600E inhibitor PLX4032 (RG7204). Biochemical and Biophysical Research Communications 2011404 958-962. (doi:10.1016/j.bbrc.2010. 12.088)

28 Ahmed M, Barbachano Y, Riddell A, Hickey J, Newbold KL, Viros A, Harrington KJ, Marais R \& Nutting CM. Analysis of the efficacy and toxicity of sorafenib in thyroid cancer: a phase II study in a UK based population. European Journal of Endocrinology 2011 165 315-322. (doi:10.1530/EJE-11-0129)

29 Hoftijzer H, Kapiteijn E, Schneider TC, Hovens GC, Morreau H, Gelderblom $\mathrm{H} \&$ Smit JWA. Tyrosine kinase inhibitors in differentiated thyroid carcinoma: a review of the clinical evidence. Clinical Investigation 20111 241-252. (doi:10.4155/cli.10.29)

30 Yang L, Shi L \& Fu Q. Efficacy and safety of sorafenib in advanced renal cell carcinoma patients: results from a long-term study. Oncology Letters 20123 935-939.

31 Vincenzi B, Santini D, Russo A, Addeo R, Giuliani F, Montella L, Rizzo S, Venditti O, Frezza AM, Caraglia M, Colucci G, Del Prete S \& Tonini G. Early skin toxicity as a predictive factor for tumor control in hepatocellular carcinoma patients treated with sorafenib. Oncologist 201015 85-92. (doi:10.1634/theoncologist.2009-0143)

32 Kapiteijn E, Schneider TC, Morreau H, Gelderblom H, Nortier JW \& Smit JW. New treatment modalities in advanced thyroid cancer. Annals of Oncology 201223 10-18. (doi:10.1093/annonc/mdr117)

Received 9 May 2012

Revised version received 14 August 2012

Accepted 22 August 2012 